\title{
Uptake of screening for breast cancer in South Lancashire
}

\author{
A Gatrell ${ }^{1}, \mathrm{~S}$ Garnett ${ }^{2}, \mathrm{~J}$ Rigby ${ }^{3}, \mathrm{~A} \mathrm{Maddocks}^{3}$ and M Kirwan ${ }^{3}$ \\ ${ }^{1}$ Institute for Health Research, Lancaster University, Alexandra Square, Lancaster, LA1 4YT; ${ }^{2}$ Department of Public \\ Health, South Lancashire Health Authority, Eccleston, Lancashire, PR7 5PD; and ${ }^{3}$ North West Regional Research \\ Laboratory, Department of Geography, Lancaster University, Lancaster, LA1 $4 Y B$
}

\begin{abstract}
Study objective: To describe and explain variation among general practices in the uptake of screening for breast cancer.

Design: Analysis of the variation in uptake of screening by general practice. Uptake rates are calculated and related to a social deprivation score created for each practice, and to the presence of at least one female general practitioner. Setting: South Lancashire Health Authority, England.

Patients: All women aged 50-64 y registered with Lancashire Family Health Services Authority and resident in South Lancashire in $1988-1995$.

Main results: Variation in the uptake of screening for breast cancer during Round 1 of the national programme is explained partly by a deprivation score for each practice and by the presence of at least one female general practitioner. In Round 2 the deprivation index continues to explain variation, but the effect of a female GP has diminished. The number of hours worked by practice nurses has no effect on uptake of breast screening.

Conclusions: Variation in the uptake of breast cancer screening is closely related to social deprivation. Results suggest that the presence of a female general practitioner has a beneficial effect on uptake.
\end{abstract}

Keywords: breast cancer; screening; uptake rates; social deprivation; South Lancashire

\section{Introduction}

In public health there is a continuing need for baseline information concerning the uptake of preventative services, such as immunisation, and screening for cervical and breast cancer. Those engaged in purchasing or commissioning health care need to describe and understand patterns of variation in screening uptake, as well as outline and evaluate policies that will increase the uptake of preventative health care. Such requirements are given fresh impetus by contemporary concerns with meeting Health of the Nation targets ${ }^{1}$ and with patterns of health variations. ${ }^{2}$ Any inequalities in the uptake of preventative services could conceivably influence the pattern of disease and disease survival rates across social groups. ${ }^{3}$

One important approach to monitoring such variation is to prepare 'equity audits', analysing measures of need, access and outcome according to demographic characteristics and by socio-economic profiles of area of residence. ${ }^{4}$ In particular, postcoded information on uptake can be linked with Enumeration District data from the 1991 Census $^{5,6}$ in order to monitor levels of uptake of services. This study outlined below considers spatial variations in the pattern of uptake, at the GP practice level, for breast cancer screening, focusing on South Lancashire Health Authority, an area covering three Local Authority Districts (West Lancashire, Chorley, and South Ribble) (Figure 1). Although breast screening is not performed in the GP surgery it is possible, as we show below, to describe variations in uptake at the practice level.

Describing, accounting for, and seeking to address, spatial variation in uptake of preventative services such as screening for breast cancer is therefore an important area of research in public health medicine. Description calls for the accurate recording of screening status, with locations given

Correspondence: Prof AC Gatrell.

Accepted 13 May 1998 by unit postcodes. ${ }^{7}$ Explanation requires links to be built to other sources of data, ${ }^{5,6}$ notably that relating to Census areas and GP practices. Policy intervention demands an indication of where the poorly performing areas or practices are located, and seeing whether mobile health facilities might be better located, and if so where. And a major goal in public health medicine is to see that any inequalities in access to health care are removed or at least diminished.

Slippery though the concept of social deprivation is, it is generally recognised that it has several dimensions: thus requiring more than one variable to characterise it adequately. Two indices in common use within the health services $^{8}$ are those of Jarman and Townsend. ${ }^{9}$ However, more recently, Carstairs, ${ }^{10}$ working in Scotland, has derived an index which although it has much in common with that of Townsend, correlates slightly better ${ }^{11}$ with measures of mortality and morbidity. The variables are, in full: overcrowding (people in private households living at a density of $>1$ person per room as a proportion of all people in private households); male unemployment (proportion of economically active males who are seeking work); no car (proportion of all people in private households with no car); and low social class (proportion of all people in private households with head of household in social class 4 or 5). A Carstairs score is derived as an unweighted summation of these four variables, after standardising them (subtracting the mean from each proportion and dividing by the standard deviation).

We wished to assess the extent to which both an appropriate measure of social deprivation and characteristics of GP practices could explain variations in uptake for breast screening. Among the latter, we hypothesise that the presence of a woman doctor in the practice has an impact on screening, even though the screening is not itself undertaken in the practice. Research into women's preferences for male and female GPs indicates that, where there is a choice it is for a female GP, particularly where 


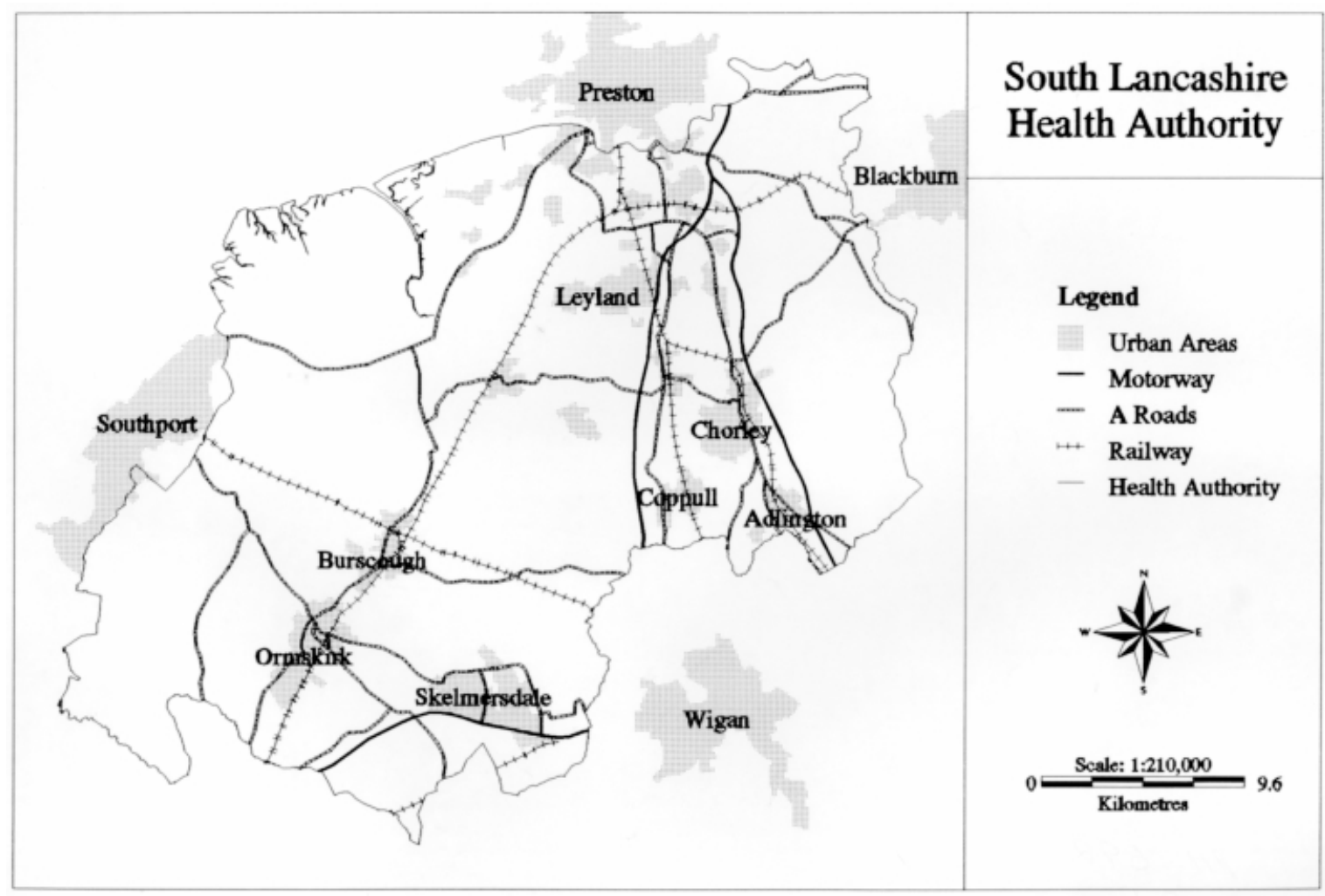

Figure 1 Study area.

the problem is considered embarrassing. ${ }^{12-14}$ We also consider whether the role of nurses within the practice, as measured by the hours worked by such staff, had any impact on screening uptake.

\section{Material and methods}

Postcoded data were obtained from Lancashire Family Health Services Authority, on all women who were eligible for breast screening and aged 50-64y. Approximately 24000 such women resident in South Lancashire were available for screening during Round 1 (January 1989February 1992) and Round 2 (March 1992-June 1995). Data included a code for the General Practitioner, date of birth, full unit postcode, and details of screening status. The postcode-ED directory ${ }^{5}$ was used to attach to each record an Enumeration District (ED) code relating to the 1991 Census.

The 1991 Census data were extracted for all EDs in the area for which South Lancashire Health Authority was responsible. The Carstairs index was computed for each ED and a Carstairs 'score' attached to each record of the database (as described in Reference ${ }^{6}$, for example). For the set of women registered with a particular GP practice it was possible to compute an average Carstairs score and attach this to each practice as a measure of social deprivation. Clearly, in the absence of a complete patient register it is impossible to define a deprivation score representative of the practice population as a whole (as done in several recent studies; see References 15 and 16, for example).

Attributes attached to the database of practices in South Lancashire included the practice code and name, the screening uptake rate for Round 1 and Round 2, the number of women invited during each round, the number of female GPs in each practice, whether or not the practice was single-handed, and the total number of hours worked by nurses in each practice. Information concerning practice characteristics was taken from FHSA Medical Lists, prepared in 1995.

Regression analysis sought to explain variation in uptake, by practice (the dependent or response variable), using Census-based measures and practice characteristics as covariates. Since the number of women screened is a proportion of the number of women invited to attend, an appropriate regression model is a logistic model, which was fitted using GLIM. ${ }^{17}$

\section{Results}

Practices varied considerably in the total number of women invited for screening, and analysis was conducted only on practices where at least 50 women were invited. Mean uptake for the 57 practices for which data for Round 1 were analysed was $82.9 \%$ (range $=57.0-95.5 \%$ ). In Round 2, mean uptake was $83.9 \%$ for the 60 practices where over 50 women were invited for screening (range $=64.8-93.3 \%$ ).

A plot of percentage uptake during Round 1 against the Carstairs index reveals a clear inverse relationship, with uptake declining as social deprivation increases (Figure 2). In general, practices which had no female GP performed worse, regardless of deprivation score. Confirmation of the importance of both deprivation levels and prescence of a female GP is given by a logistic regression analysis (Table 1), which shows that both the deprivation score and the presence of a female GP are highly significant predictors of screening uptake. Social deprivation reduces the odds of uptake by $9 \%$, while the presence of a female GP increases it by $44 \%$. 


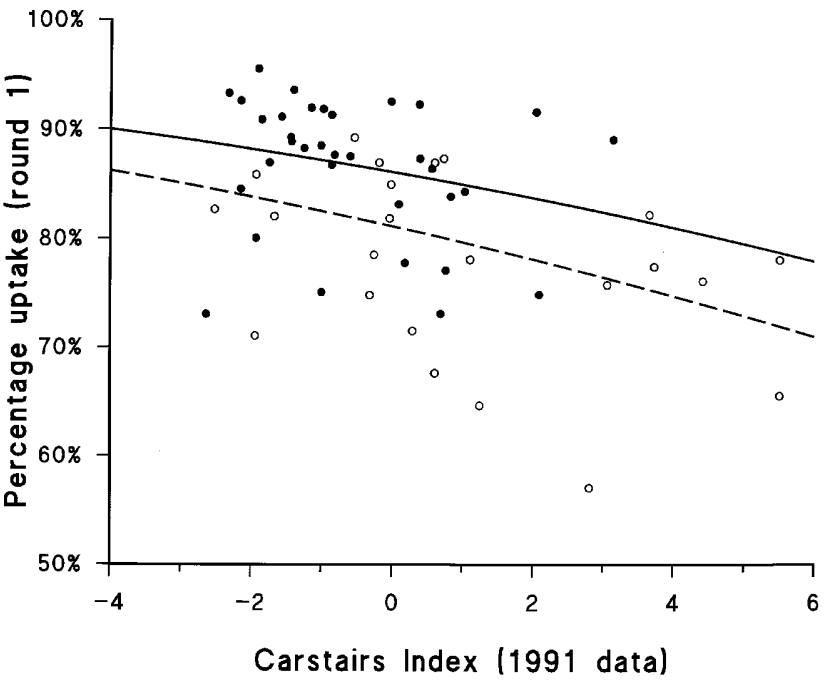

Figure 2 Percentage screening uptake (Round 1) in relation to deprivation (Carstairs score) and presence/absence of female GP (solid circles denote practices with at least one female GP; solid line is logistic regression curve for such practices).

Results for Round 2 of the screening programme are broadly comparable (Figure 3; Table 1), although the importance of social deprivation increased (a $12 \%$ reduction in the odds of uptake) while that of female GPs has waned (an $11 \%$ increase in the odds of uptake). Both variables continue to have a statistically significant effect on uptake rates. No interaction effects were observed between the two covariates.

For both Rounds 1 and 2 these models were the best that could be fitted to the data. Other variables failed to add any explanatory power. There was no relationship between uptake and hours worked by practice nurses. There was no relationship between uptake of screening and whether or not the practices were single-handed. The influence of ethnicity in this geographical setting is negligible, since only $0.11 \%$ of the female population aged $50-64 \mathrm{y}$ is of Asian background (compared with $1.52 \%$ nationally).

\section{Discussion}

Our results suggest that uptake rates, for breast screening at practice level, are in part influenced by the socio-economic make-up of the areas from which patients are drawn; as well as by whether or not the practice has at least one female practitioner. It should be noted that the measures of practice characteristics (simply presence or absence of female GP and of practice nurses) are very crude. Further research to assess the hours they work (which might vary from a few hours per week to full-time) might be useful. That the presence of a practice nurse has no impact on uptake is not surprising since the screening does not take

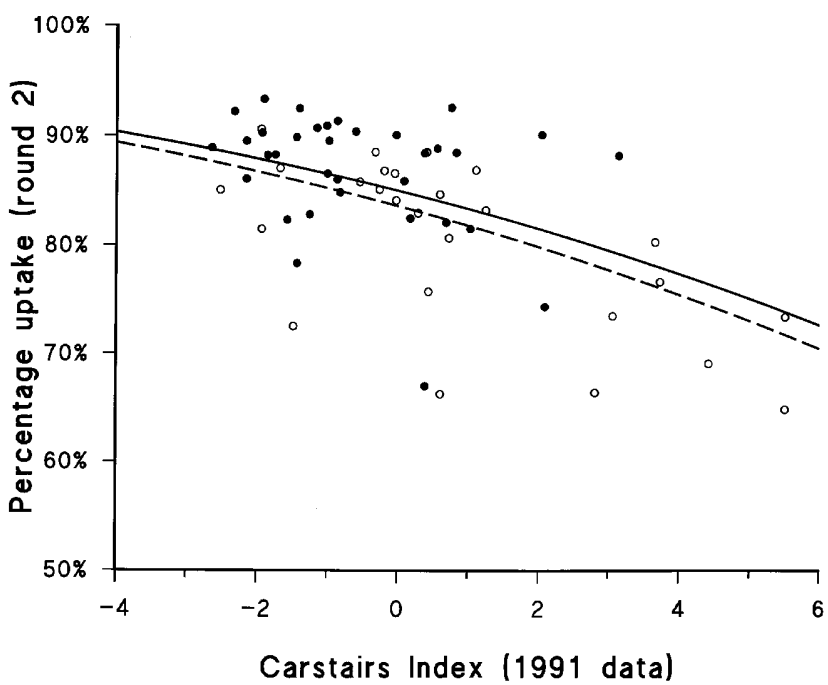

Figure 3 Percentage screening uptake (Round 2) in relation to deprivation (Carstairs score) and presence/absence of female GP (solid circles denote practices with at least one female GP; solid line is logistic regression curve for such practices).

place at the surgery; in addition, practice nurses may be seen for quite specific problems and opportunities for discussion of breast screening might not present themselves. The separation of the screening from the practice surgery means that it may be surprising to detect an influence relating to the practice having a female GP, but evidence from our study suggests there is an effect. This parallels findings from a study of cervical smear uptake rates in part of London, ${ }^{18}$ a context in which there is, understandably, an increased uptake in practices with a female general practitioner. We speculate that, during consultations with a female general practitioner, a patient nearing the date at which she is likely to be called for breast examination may well be encouraged to attend; such encouragement may, in certain circumstances, be more likely to emerge from a female General Practitioner than a male one. Earlier research ${ }^{12,14}$ has shown that female GPs are considered to be easier to relate to and to show more understanding of women's health problems. Research has also indicated ${ }^{19}$ that lack of access to a female GP may be a barrier to preventive health care, especially in deprived areas, where the proportion of female GPs is often small. However, we detected no interaction between the deprivation variable and presence of female doctors.

The kind of analysis reported here permits a public health doctor to discuss with primary health care colleagues reasons for low uptake, having already 'ruled out' the impact of local contextual factors such as material deprivation. It is important to be able to do this. Studies at the level of administrative units such as electoral wards are all very well, but electoral wards cannot be encouraged to improve their performance; GPs, on the other hand, can.

Table 1 Logistic regression analysis of uptake of breast cancer screening

\begin{tabular}{lcccccr}
\hline & Round 1 & & & \multicolumn{2}{c}{ Round 2 } \\
\cline { 1 - 1 } Variable & Odds ratio & Confidence interval & & Variable & Odds ratio & Confidence interval \\
\hline Deprivation score & 0.91 & $(0.89-0.93)$ & & Deprivation score & 0.88 & $(0.86-0.90)$ \\
Female GP & 1.44 & $(1.26-1.58)$ & & Female GP & 1.11 & $(1.02-1.21)$ \\
\hline
\end{tabular}


'Raw' league tables of practice performance, unadjusted for contextual effects, may be misleading. Attention might be directed to practices (of which there are several in this study area) where screening uptake is poor despite low deprivation scores. ${ }^{20}$ We have demonstrated that this is in part a function of their lack of a female GP; but analysis of the residuals indicates practices which are performing poorly regardless of deprivation and GP gender mix. Whether these variations in performance reflect other unmeasured features of the GP catchments, or the perceived quality of the service offered by GPs (their friendliness, approachability, quality of waiting rooms and so on), or local cultures (affecting women's own perceptions, attitudes and concerns about screening, for example) is something which only more detailed and perhaps qualitatively-based research can uncover. There may, in addition, be organisational or administrative changes to the screening programme that might be made; for example, asking GPs to sign the first reminder letters may produce better uptake than more anonymous reminders sent from the screening units.

The mean uptake rates reported here compare very favourably with those elsewhere. For example, Majeed et $a l^{21}$ reported a screening rate of $58.2 \%$ in Round 1 for practices in Merton, Sutton and Wandsworth FHSA (compared with $83 \%$ in South Lancashire). They too find inverse correlations with a measure of social deprivation (proportion of households lacking a car), but also with ethnicity (percentage non-white population). Rates were significantly higher in practices which were computerised, while the size of practice (number of partners) had a significant positive effect in predicting screening rates over the set of 131 practices considered. In contrast to the results reported above there was no significant difference in screening rates between practices with and without a female partner. In survey research conducted in inner London, Sutton et $a l^{22}$ have suggested that indicators of social class and education do not predict attendance, although they find that women living in rented accommodation are less likely to attend for screening.

We need to recognise the limitations of available data, particularly those relating to the screening programme. FHSA registers may be incomplete or inaccurate; they may be subject to 'list inflation'. A proportion of postal invitations will not reach those women to whom they are directed. A study in London ${ }^{21}$ suggested that list inflation correlated significantly with screening rates. No data were available to us to test this proposition locally, but there is no reason to believe that the problem is especially severe in south Lancashire or that it has introduced serious biases into the analysis.

We have assumed that practice characteristics (notably, presence of at least one female GP) remain constant over the period of study, using information available at a single point in time. Of course, General Practitioners and practices are not static in composition. In addition, there is no easy way of knowing whether a practice is employing a female GP on a sessional basis.

While EDs and electoral wards are spatially welldefined, GP catchments do not follow lines which can be drawn on a map. Patients may come from different parts of a Health Authority's jurisdiction, with some practices drawing patients from a restricted geographical area whilst others may have patients who are dispersed over a wider area. This renders the task of defining 'catchment' characteristics, fraught with difficulty, and whilst some attempts have been made to do this ${ }^{15}$ and others ${ }^{6,16}$ have reviewed alternative methods, there is no simple solution. However, in the absence of data relating to individuals' access to material resources, matching individuals to Census Enumeration Districts is probably the best substitute measure, and is certainly an improvement on assigning postcodes to less homogeneous electoral wards. ${ }^{6,20}$ But it does lay us open to committing the ecological fallacy, where inferences about individuals are made, possibly quite incorrectly, on the basis of aggregate areal data.

\section{Conclusion}

We have demonstrated that the presence of at least one female GP has a significant impact on uptake, although this was less so during Round 2 of the national programme. The availability of practice nurses had no effect on uptake. However, the relationship between the presence of a female GP and increased uptake of screening is of interest, suggesting that perhaps during other consultations such GPs may give encouragement to women of late middle age to attend for breast screening. Evidence from Northern Ireland ${ }^{23}$ suggests that the impact of GPs in advocating the merits of mammography can have a beneficial impact on screening rates. Given extensive survey data we can begin to gain a deeper understanding of the reasons underlying variations in screening uptake, including the perceived behaviour and attitudes of screening staff, and the perceived quality of screening facilities. ${ }^{24}$ As the drive to seek further reductions in breast cancer incidence continues, such research may well pay dividends. Such research is also essential if health inequalities are to be reduced. While there is some evidence of a lower incidence of breast cancer among women in social classes 4 and 5 there is also evidence that survival from such cancers is worse in such groups. One reason, which our study supports, is that they may make less use of screening programmes, and present later. A commitment to reducing inequalities, therefore, means implementing whatever policies we can to improve screening uptake among those from poorer groups.

\section{Acknowledgements}

We thank Sally Hollis, Lecturer in Medical Statistics, Lancaster University, for her comments on a draft of this paper.

\section{References}

1 Department of Health. The Health of the Nation. Key Area Handbook: Cancer. Department of Health: London, 1993

2 Department of Health. Variations in health: What Can the Department of Health Do? Variations Sub-Group of the Chief Medical Officer's Health of the Nation Working Group. Department of Health: London, 1993.

3 Benzeval M, Judge K, Whitehead M. Tackling Inequalities in Health: An Agenda for Action: King's Fund: London, 1995.

4 Majeed FA, Chaturvedi N, Reading R, Ben-Shlomo Y. Monitoring and promoting equity in primary and secondary care. $B M J$ 1994; 308: $1426-1429$. 
5 Majeed FA, Cook DG, Poloniecki J, Martin D. Using data from the 1991 Census. BMJ 1995; 310: 1511-1514.

6 Scrivener G, Lloyd DECF. Allocating census data to general practice populations: implications for study of prescribing variation at practice level. $B M J$ 1995; 311: $163-165$.

7 Gatrell AC, Dunn CE, Boyle PJ. The relative utility of the Central Postcode Directory and Pinpoint Address Code in applications of geographical information systems. Environ Plan A 1991; 23: 1447-1458.

8 Senior ML. Deprivation payments to GPs: not what the doctor ordered. Environ Plan C 1991; 9: 79-94.

9 Townsend P, Phillimore P, Beattie A. Health and Deprivation: Inequality and the North. Croom Helm: London, 1988.

10 Carstairs V, Morris R. Deprivation and Health in Scotland. Aberdeen University Press: Aberdeen, 1991

11 Morris R, Carstairs V. Which deprivation? A comparison of selected deprivation indexes. J Public Health Med 1991; 13: $318-326$.

12 Nichols S. Women's preferences for sex of doctor: a postal survey. J Roy Coll General Pract 1987; 37: 540-543.

13 Waller K. Women doctors for women patients? $\mathrm{Br} J$ Med Psychol 1988; 61: 125-135.

14 van den Brink-Muinen A, de Bakker DH, Bensing JM. Consultations for women's health problems: factors influencing women's choice of sex of general practitioner. $\mathrm{Br} J$ General Pract 1994; 44: 205-210.
15 Martin D, Senior ML, Williams HCWL. On measures of deprivation and the spatial allocation of resources for primary health care. Environ Plan A 1994; 26: 1911-1929.

16 Haynes RM et al. Evaluation of methods for calculating census health indicators for GP practices. Public Health 1995; 109: $369-374$.

17 Aitkin M, Anderson D, Francis B, Hinde J. Statistical Modelling in GLIM. Oxford University Press: Oxford, 1989.

18 Majeed FA et al. Using patient and general practice characteristics to explain variations in cervical smear uptake rates. $B M J$ 1994; 308: $1272-1276$.

19 Cooke M, Ronalds C. Women doctors in an urban general practice. $B M J$ 1985; 290: 753-761.

20 Majeed FA et al. Sociodemographic variables for general practices: use of census data. $B M J$ 1995; 311: $163-165$.

21 Majeed FA et al. Do general practitioners influence the uptake of breast cancer screening? J Med Screen 1995; 2: $119-124$.

22 Sutton S, Bickler G, Sancho-Aldridge J, Saidi G. Prospective study of predictors of attendance for breast screening in inner London. J Epidemiol Community Health 1994; 48: 65-73.

23 Kee F. Do general practitioners facilitate the breast screening programme? Eur J Cancer Preven 1992; 1: 231-238.

24 Vaile MS, Calnan M, Rutter DR, Wall B. Breast cancer screening services in three areas: uptake and satisfaction, J Public Health Med 1993; 15: 37-45. 